\title{
Analyzing dendritic growth in a population of immature neurons in the adult dentate gyrus using laminar quantification of disjointed dendrites
}

\author{
Shira Rosenzweig* and J. Martin Wojtowicz \\ Department of Physiology, University of Toronto, Toronto, ON, Canada
}

\section{Edited by:}

Silvia De Marchis, University of Turin, Italy

\section{Reviewed by:}

Heather A. Cameron, National Institutes of Health, USA

Sophie Tronel, Institut National de la

Santé et de la Recherche Médicale, France

\section{*Correspondence:}

Shira Rosenzweig, Department of Physiology, University of Toronto, 1 King's College Circle, Toronto, ON, Canada M5S 1 A8.

e-mail: s.rosenzweig@utoronto.ca
In the dentate gyrus (DG) of the hippocampus, new granule neurons are continuously produced throughout adult life. A prerequisite for the successful synaptic integration of these neurons is the sprouting and extension of dendrites into the molecular layer of the DG. Thus, studies aimed at investigating the developmental stages of adult neurogenesis often use dendritic growth as an important indicator of neuronal health and maturity. Based on the known topography of the DG, characterized by distinct laminar arrangement of granule neurons and their extensions, we have developed a new method for analysis of dendritic growth in immature adult-born granule neurons. The method is comprised of laminar quantification of cell bodies, primary, secondary and tertiary dendrites separately and independently from each other. In contrast to most existing methods, laminar quantification of dendrites does not require the use of exogenous markers and does not involve arbitrary selection of individual neurons. The new method relies on immunohistochemical detection of endogenous markers such as doublecortin to perform a comprehensive analysis of a sub-population of immature neurons. Disjointed, "orphan" dendrites that often appear in the thin histological sections are taken into account. Using several experimental groups of rats and mice, we demonstrate here the suitable techniques for quantifying neurons and dendrites, and explain how the ratios between the quantified values can be used in a comparative analysis to indicate variations in dendritic growth and complexity.

Keywords: dendrites, adult neurogenesis, dentate gyrus, doublecortin, dendritic complexity

\section{INTRODUCTION}

Laminar arrangement of neuronal fields and axonal projections is a characteristic of the mammalian forebrain. This anatomical feature is particularly prominent in the dentate gyrus (DG) of the hippocampal formation, where incoming afferents make synaptic contacts with granule neurons within the three laminar regions of the molecular layer (ML; Ruth et al., 1982; Witter, 2007). In addition to the layering of the afferent inputs which has a well-known topographical and physiological significance, the cell bodies of the granule neurons are similarly arranged in layers and their position is related to their chronological formation (Wang et al., 2000). A consequence of such laminar arrangement is an intricate matrix of connections between the upstream entorhinal cortex and the first relay of the tri-synaptic circuit of the hippocampus.

Into this complex circuitry there is a continuous introduction of new neurons born in the sub-granular zone (SGZ) of the DG throughout adult life (Christie and Cameron, 2006). Since neuronal progenitors are mostly devoid of any neural processes, a fundamental milestone in the development of adult-born neurons is the extension of dendrites into the ML where the synaptic connections are formed (Carlen et al., 2002; Abrous et al., 2005). Because this progression is critical for successful neuronal integration and

Abbreviations: DCX, doublecortin; DG, dentate gyrus; GCL, granule cell layer; GFP, green fluorescent protein; ML, molecular layer; SGZ, sub-granular zone. subsequent functionality, studies evaluating adult neurogenesis often include measurements of dendritic growth in developing neurons and use these measurements as indicators of the growth process (Eadie et al., 2005; Rao et al., 2005; Overstreet-Wadiche et al., 2006; Redila and Christie, 2006; Tronel et al., 2010; RamirezRodriguez et al., 2011).

Most existing methods used to measure dendritic length and complexity require the complete visualization of isolated neurons and a subsequent tracing of their entire dendritic tree. In these instances the neurons are either loaded with a dye (e.g., Golgi staining) or induced to express a fluorescent protein which is introduced by a viral vector or through genetic manipulation (Zhao et al., 2006; Nishi et al., 2007; Ide et al., 2008; Ambrogini et al., 2010; Winner et al., 2011). While these methods produce an accurate analysis on a single-cell resolution, extrapolating the acquired data to a larger neuronal population might prove inaccurate if the staining technique selectively labels only specific neurons. Additional selection bias might also occur in these cases if the researcher chooses to measure "convenient" cells which are visualized more clearly and without overlap with other neurons.

To overcome such problems we delineate here a new approach for analyzing dendritic growth in a sub-population of adult-born neurons in the DG. We demonstrate how laminar quantification of disjointed dendrites accurately represents all the cells in the population and does not require the use of exogenous markers or dyes. The neurons are identified by their expression of endogenous 
markers such as doublecortin (DCX) or PSA-NCAM, which are well-established and frequently used in neurogenesis research to identify and quantify immature neurons.

Doublecortin is a microtubule-associated protein required for neuronal migration and differentiation (Gleeson et al., 1999). In the adult DG DCX is expressed throughout the soma and neurites of immature neurons residing in the SGZ and inner granule cell layer (GCL). DCX expression begins shortly after the neurons are born, persists for approximately 2 weeks in rats and 3 weeks in mice, and then gradually declines as the neurons continue to mature (Rao and Shetty, 2004; Snyder et al., 2009). Due to a similar expression pattern in the DG, this marker can be complemented or interchanged by PSA-NCAM, a polysialylated form of the neural cell adhesion molecule which has been shown to be involved in the regulation of myelination in the central nervous system as well as axonal guidance and synapse formation (Seki and Arai, 1999; Nguyen et al., 2003).

While both markers are expressed throughout the cell bodies and neurites, it is often difficult to visualize the cells that express them in their entirety due to overlap between adjacent cells. This may prevent the researcher from accurately discerning which dendrites originate from which cells. In addition, neurites are often separated from their cell bodies when the tissue is sectioned, resulting in either "orphaned" dendrites or cell bodies that appear to have no dendrites, but actually have well-developed dendritic trees which now reside in an adjacent section.

These pitfalls have minimal or no effect when using laminar quantification of disjointed dendrites, because this method does not require the visualization of any vertical connections (i.e., between cell bodies and dendrites, or primary dendrites and secondary/ tertiary dendrites). Instead, the method relies on the known alignment of immature neurons in the DG (cell bodies in the SGZ, dendrites extended vertically toward the ML, see Figure 1) to quantify cell bodies and dendrites in a disjointed manner - separately and independently from each other. The ratios between the acquired

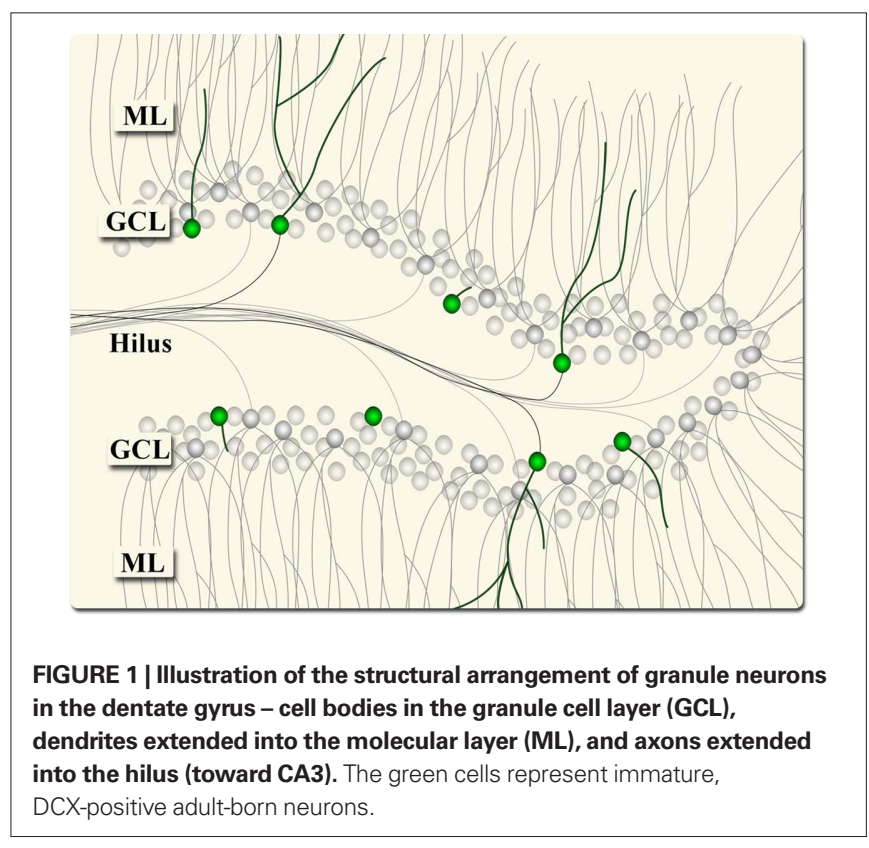

numbers can be used in a comparative analysis to indicate changes in the number of cells and the extent of dendritic arborization in the population. We explain here how several visualization techniques can be used to quantify cells and dendrites with or without the acquisition of high-resolution stack confocal images, and demonstrate how the calculated ratios change between several experimental groups. In order to highlight the advantages of our method, we also compare it to the more commonly used tracing technique.

\section{MATERIALS AND METHODS ANIMALS AND TREATMENTS}

For demonstration purposes we used tissue taken from two groups of 4-months-old male Sprague-Dawley rats (Charles River, Quebec), and two groups of 3-months-old male mice. Each of the rat groups included four animals: the experimental (treated) group was administered seven daily injections (intra-peritoneal) of a drug known to modulate GABA-ergic neurotransmission, while the control group was injected with saline. The animals were sacrificed 2 weeks after the last injection. One additional group of 3-months-old rats (Sprague-Dawley) was used for retrovirus-mediated green fluorescent protein (GFP) labeling. The mouse groups were consisted of four wild-type C57BL/6J mice (WT group), and four knock-out mice lacking a specific ionotropic GABA receptor subunit (KO group). All animal procedures conformed to animal health and welfare guidelines of the University of Toronto.

\section{RETROVIRUS-MEDIATED LABELING OF NEW NEURONS IN RATS}

The animals were anesthetized using intra-peritoneal injection of ketamine/xylazine $(85 / 5 \mathrm{mg} / \mathrm{kg})$, and then placed in a stereotaxic device. A retroviral vector expressing GFP was originally donated by Dr. F. Gage's laboratory (Salk Inst., San Diego; van Praag et al., 2002) with additional batches of the virus produced locally at the University of Toronto in collaboration with Dr. C. Morshead (Medicine). A concentrated solution of the virus $\left(>10^{8}\right.$ infectious units $/ \mathrm{ml}$ ) was prepared and injected into the DG at the following coordinates (two sites in each DG, $1.5 \mu \mathrm{l} /$ site): AP: $-3.3, \mathrm{ML}: \pm 1.5$, DV: -4.4 , and AP: -4.3 , ML: $\pm 2.6, \mathrm{DV}:-4$. The animals were sacrificed 2 weeks after the injection.

\section{IMMUNOHISTOCHEMISTRY}

Animals were perfused intracardially with phosphate-buffered saline followed by $4 \%$ paraformaldehyde. The brains were removed and fixed in $4 \%$ paraformaldehyde for $24 \mathrm{~h}$ at $4^{\circ} \mathrm{C}$. Hemispheres were sectioned using a vibratome into $40 \mu \mathrm{m}$ coronal sections, yielding approximately 100 sections along the hippocampus in rats and 60 in mice. For DCX immunostaining, nine sections were sampled at fixed intervals from each animal. Free-floating sections were incubated with a primary goat anti-DCX antibody (1:200, Santa Cruz Biotechnology, $24 \mathrm{~h}$ at $4^{\circ} \mathrm{C}$ ), followed by Alexa 488 donkey anti-goat secondary antibody (1:200; Invitrogen; 2 h at RT). All antibodies were diluted in phosphate-buffered saline containing $0.03 \%$ Triton X-100.

\section{IMAGING AND TRACING OF GFP-POSITIVE NEURONS}

Sections from animals injected with GFP-expressing retrovirus were imaged with a Leica TCS-SL confocal microscope (Leica Microsystems) using a $40 \times$ oil immersion objective lens. The 
analysis of GFP-positive neurons was performed in maximumintensity projection of a $z$-series stack acquired at $0.8 \mu \mathrm{m}$ intervals throughout $40 \mu \mathrm{m}$ thick sections. For the demonstrated comparison we chose stacks that contained no GFP-positive cell bodies within $10 \mu \mathrm{m}$ of the surface of the section, to minimize the inclusion of cells with cut-off dendritic trees. The dendrites in the image were traced using NeuronJ (Meijering et al., 2004) and the number of branching points was counted manually from the same image. Similar technique has been described previously in numerous studies (Overstreet-Wadiche et al., 2006; Zhao et al., 2006; Winner et al., 2011).

\section{IMAGING AND QUANTIFICATION OF DCX-POSITIVE CELLS AND DENDRITES}

The analysis of dendrites in DCX-positive cells was performed both under a fluorescent microscope (Nikon Optiphot-2) using a 40× objective lens and in images obtained with a Leica TCS-SL confocal microscope (Leica Microsystems) using a $40 \times$ oil immersion objective lens. Each image was constructed from a maximum-intensity projection of a $z$-series stack acquired at $0.8 \mu \mathrm{m}$ intervals throughout $40 \mu \mathrm{m}$ thick sections. While it is not necessary to reconstruct an entire section into a single image for the quantification, the DG area should be imaged with minimum gaps or overlap while making sure the gain settings remain constant between images.

The typical arrangement of DCX-positive cells in the DG (as illustrated in Figure 1), mandates that the cell bodies be counted in the SGZ and inner GCL, the primary dendrites counted in the middle of the GCL, secondary dendrites counted along the external edge of the GCL and tertiary branches counted in the ML. The cell bodies and dendrites are quantified separately and several independent values are obtained. The first value $(a)$ represents the number of DCX-positive cells and is traditionally obtained by visualizing and counting the cell bodies under a fluorescent microscope, a technique which can be employed in this case as well. Alternatively, when confocal stack images are obtained for further dendritic analysis, the cell bodies can be quantified as they appear in the images. For example, the estimated number of cells in Figure 2A is 10.

The next several values $(b, c, d)$ represent the number of dendrites at distinct spans from the cell bodies layer. Theoretically, while three values may be sufficient, if additional values are collected more information can be inferred regarding the dendritic complexity of the cells. The first value in this series $(b)$ should represent the number of primary dendrites and thus be measured just above the cell bodies of DCX-positive cells (approximately in the middle of the GCL). Line 1 in Figure 2A demonstrates the correct area for the quantification of $b$. The dendrites along the line can be quantified by manually tracing the line and marking any dendrites along its path, or by using image processing software such as ImageJ (NIH) to plot a profile of the intensity of the signal along the line. The profile of line 1 is displayed in Figure $\mathbf{2 B}$, and a threshold of 7 was set to yield a correct estimation of 13 dendrites (peaks). The same threshold was subsequently used for the next two values in the series; however, since the brightness of tertiary dendrites may differ from that of primary dendrites, and the levels of background "noise" may differ between the GCL and ML, a different threshold will occasionally have to be set for each measurement line.

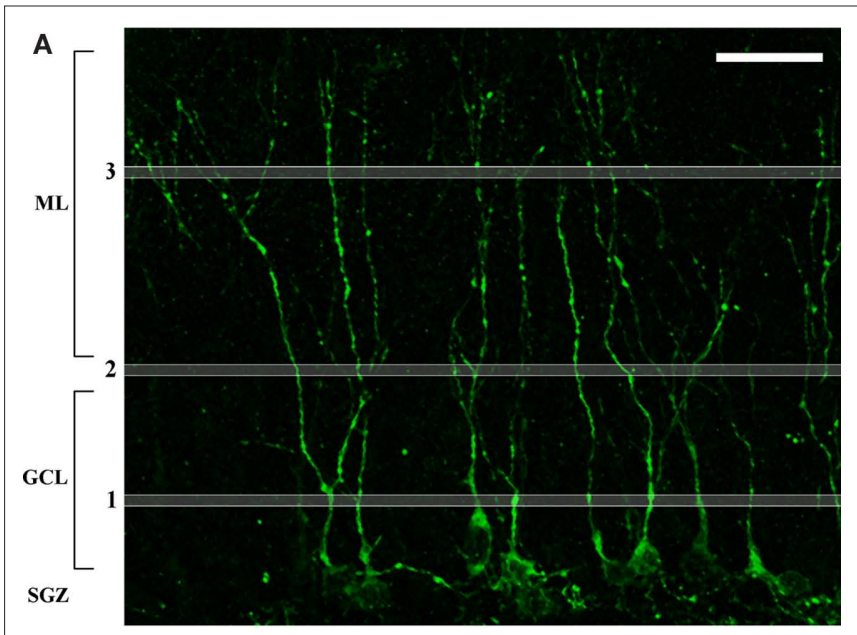

B

Primary dendrites ( line 1)

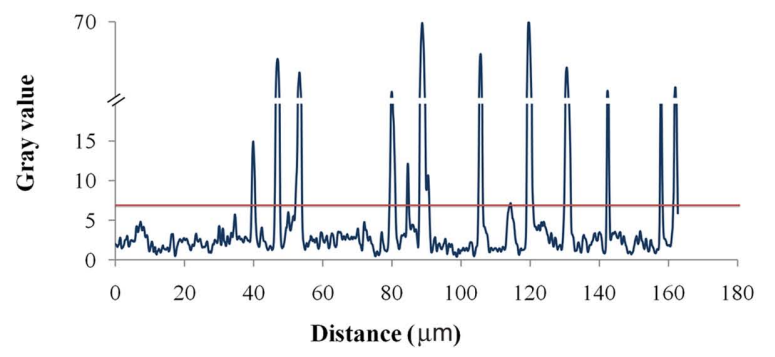

C

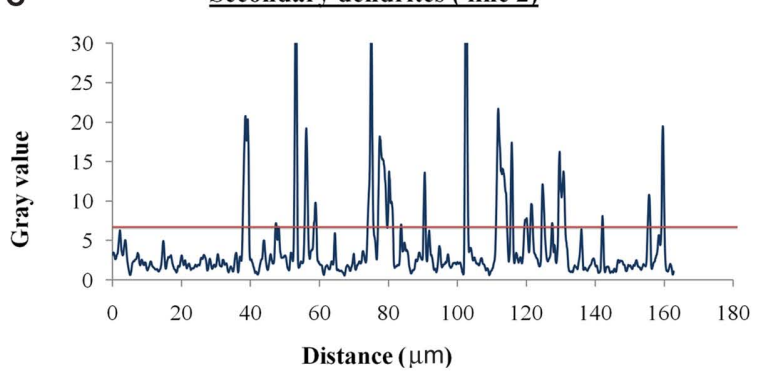

D

$\underline{\text { Tertiary dendrites ( line 3) }}$

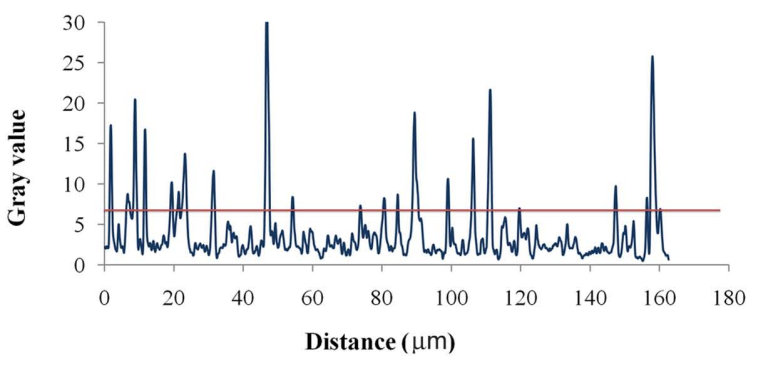

FIGURE 2 | Example of dendritic quantification. (A) High-resolution confocal stack image of a section through the DG immunostained for DCX. DCX-positive cell bodies are arranged along or just above the SGZ, primary dendrites are quantified along line 1 in the middle of the GCL, secondary dendrites are quantified along line 2 on the outer edge of the GCL and tertiary dendrites are quantified along line 3 in the ML. Scale bar $=20 \mu \mathrm{m}$ (B-D) plot profiles for lines 1-3 obtained using ImageJ software. The red line indicates the threshold (set for 7) and all the peaks that cross it are counted. 
The value $c$ represents secondary dendrites and is measured along the outer edge of the GCL (line 2 in Figure 2A). The number of dendrites "encountered" along the line is measured similarly to line 1. The profile of line 2 is displayed in Figure 2C and indicates 22 secondary dendrites for a threshold of 7 . The next value $(d)$ represents tertiary branches and will be measured in the same fashion in the ML (line 3 in Figure 2A). The profile of line 3 is displayed in Figure 2D and indicates 22 dendrites for a threshold of 7 . The locations of the three lines demonstrated here can be shifted according to the specific requirements of the experiment, and additional values/lines can be added if needed.

While this technique provides consistent results, it is also possible to perform dendritic measurements under a fluorescent microscope in the same manner as when cell bodies are conventionally counted. The three lines - middle GCL, outer edge of the GCL, and middle ML can be visualized and separately "traced" by the investigator, and any "encounter" with dendrites along the lines counted. This technique is significantly less time-consuming because it does not require the imaging of entire sections; however, the accuracy and consistency of the results may vary considerably according to the investigator's experience and skill.

\section{ANALYSIS}

Changes in the number of DCX-positive cells $(a)$ provide important information regarding variations in the rate of adult neurogenesis between different experimental groups. However, changes in the values representing dendrites $(b, c$, and $d$ ) become indicative of variations in dendritic complexity only when the ratios between the values are calculated and compared across experimental groups. For instance, a decrease in the number of primary dendrites $(b)$ would be a given in a case where the total number of cell bodies $(a)$ is decreased, because primary dendrites originate directly from the cell bodies. As long as the ratio $b / a$ is unchanged, the decrease in the number of primary dendrites would not be a sign of diminished dendritic growth. However, a decrease in the number of primary dendrites with no change in the number of cells (i.e., $b / a$ is lower), might indicate a reduction in the initial ability of young neurons to sprout dendrites. In that case the number of secondary dendrites (c) might be reduced as well (because secondary dendrites originate directly from primary dendrites), but if the effect on primary sprouting does not extend to more developed neurons (those that have already extended secondary dendrites), the ratio between tertiary and secondary dendrites $(d / c)$ will remain unchanged.

Thus, the relevant parameters are:

$N_{\mathrm{n}}$ : Number of neurons $(a)$.

$N_{\mathrm{p}}$ : The level of primary dendrite sprouting $(b / a)$.

$N_{s}$ : The level of secondary branching $(c / b)$.

$N_{\mathrm{t}}$ : The level of tertiary branching from secondary dendrites $(d / c)$.

A comparative analysis of dendritic complexity will be based on these four parameters, calculated for each experimental group.

\section{RESULTS}

\section{COMPARATIVE ANALYSIS OF DENDRITIC GROWTH IN MICE AND RATS}

We tested the laminar quantification of disjointed dendrites method in two experiments, as part of a comprehensive assessment of variations in adult neurogenesis between different groups of animals.
In experiment 1 we compared the treated rat group to the control group and in experiment 2 we compared $\mathrm{KO}$ mice to WT mice. Nine sections were sampled from each animal, imaged using a confocal microscope or analyzed using a fluorescent microscope. Only minor differences were observed between the two imaging techniques and the data displayed were obtained using the former. The number of cell bodies $(a)$, primary dendrites $(b)$ secondary dendrites $(c)$, and tertiary dendrites $(d)$ were quantified along $1 \mathrm{~mm}$ stretch in individual sections, and averaged for each animal and then for each group. Table 1 displays the averaged values $a, b, c$, and $d$ as well as the calculated ratios $N_{\mathrm{p}}, N_{\mathrm{s}}$ and $N_{\mathrm{t}}$ for each group.

The number of DCX-positive cells $\left(N_{\mathrm{n}}\right)$ was significantly higher in the treated rat group compared to the control group, as well as in KO mice compared to WT mice $(p<0.01, n=4$ for both rats and mice). The values $b, c$, and $d$ were significantly higher in the treated group and $\mathrm{KO}$ mice compared to the control group and WT mice, respectively [experiment 1 (rats): $p<0.05$ for $b, p<0.03$ for $c$ and $d, n=4$; experiment 2 (mice): $p<0.03$ for $b$ and $c, n=4$ ], with one exception ( $d$ value in mice, $p>0.07, n=4$ ). However, the calculated values $N_{\mathrm{p}}, N_{\mathrm{s}}$, and $N_{\mathrm{t}}$ revealed that the effect on the treated group in experiment 1 (rats) was different from the effect on the $\mathrm{KO}$ mice in experiment 2.

In experiment 1 , none of the three calculated ratios $N_{\mathrm{p}}, N_{\mathrm{s}}$, and $N_{\mathrm{t}}$ differed between the treated and control groups (Figure 3A, $p>0.3, n=4)$. This indicates that while the experiment induced an increase in the total number of DCX-positive cells in both species it did not affect the dendritic complexity in rats. Conversely, in experiment 2 the parameters $N_{\mathrm{s}}$ and $N_{\mathrm{t}}$ were significantly lower in the KO mice compared to the WT mice (Figure 3B, $p<0.05, n=4$ ). This indicates that the experiment induced an increase in the total number of DCX-positive cells but decreased the relative number of secondary and tertiary dendrites (even though the absolute number of secondary and tertiary dendrites, represented by the values $c$ and $d$, was higher or unchanged in the KO group - see Table 1).

\section{COMPARISON WITH THE TRACING METHOD}

In order to demonstrate the advantages of the new method over existing methods, we compared it to the commonly used dendritic tracing technique. Tracing is often used in conjunction with GFP-expressing retrovirus injection, because this labeling strategy permits better iden-

Table 1 | Summary of the data obtained for the four groups in experiments 1 and 2.

\begin{tabular}{lcccc}
\hline & Rats control & Rats treated & MiceWT & Mice KO \\
\hline$a\left(N_{\mathrm{n}}\right)$ & $84 \pm 3$ & $102 \pm 4^{* *}$ & $71 \pm 4$ & $108 \pm 9^{* *}$ \\
$b$ & $88 \pm 5$ & $112 \pm 7^{*}$ & $76 \pm 6$ & $117 \pm 10^{*}$ \\
$c$ & $121 \pm 8$ & $153 \pm 9^{*}$ & $102 \pm 6$ & $131 \pm 8^{*}$ \\
$d$ & $154 \pm 8$ & $183 \pm 8^{*}$ & $127 \pm 13$ & $132 \pm 12$ \\
$N_{\mathrm{p}}$ & $1.048 \pm 0.072$ & $1.098 \pm 0.085$ & $1.070 \pm 0.064$ & $1.083 \pm 0.058$ \\
$N_{\mathrm{s}}$ & $1.375 \pm 0.109$ & $1.366 \pm 0.116$ & $1.342 \pm 0.049$ & $1.120 \pm 0.061^{*}$ \\
$N_{\mathrm{t}}$ & $1.273 \pm 0.097$ & $1.196 \pm 0.101$ & $1.245 \pm 0.052$ & $1.008 \pm 0.066^{*}$ \\
\hline
\end{tabular}

The values a-d represent the number of cells or dendrites along $1 \mathrm{~mm}$ stretch. Each value is presented as an average of four animals $\pm S E$. The ratios $N_{p^{\prime}} N_{s^{\prime}}$ and $N_{t}$ were calculated for each animal and averaged for each group as well. ${ }^{*} p<0.05,{ }^{*} p<0.01$. 


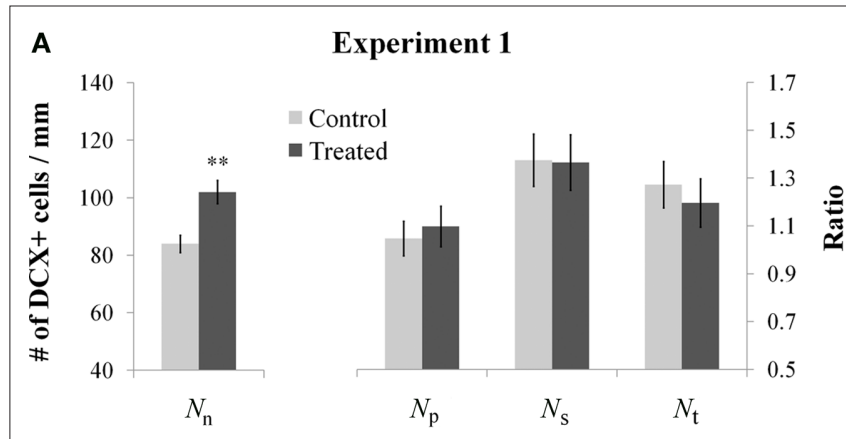

B

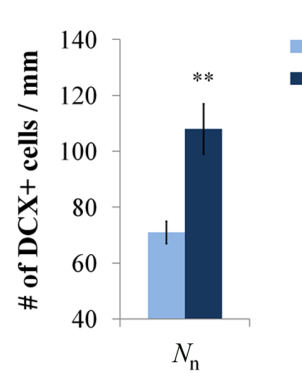

Experiment 2

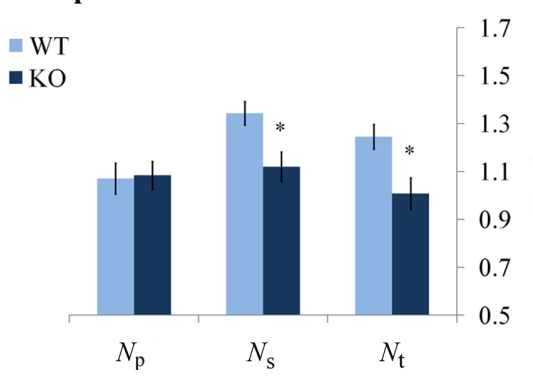

FIGURE 3 | Comparison between animal groups. (A) In experiment 1 the number of DCX-positive neurons was increased in the treated rat group with no change in dendritic complexity. (B) In experiment 2 the number of DCX-positive neurons was increased in the KO mice but secondary and tertiary branching were reduced.

tification and visualization of single-cells. (Ge et al., 2006; Zhao et al., 2006). We imaged GFP-labeled neurons in the rat DG 2 weeks after a GFP-expressing retrovirus was injected. A segment containing six GFP-positive neurons was selected for the demonstration (Figure 4A) and the dendrites in the image were analyzed using the tracing technique as well as the new laminar quantification method.

When the new method was used, "orphan" dendrites were included in the analysis (Figure 4B, blue dendrites). These dendrites were excluded during the tracing because their origin (cell body) could not be visualized. The length of the dendrites that could be traced from soma to tip (Figure 4B, black dendrites) was measured directly using the tracing method, and the complexity of the dendritic tree was evaluated by counting the number of branching points. The individual measurements as well as the averages are summarized in Figure 4C (left).

These values are different from the ones obtained using the laminar quantification method (number of neurons $-a$, primary dendrites $b$, secondary dendrites $-c$, tertiary dendrites $-d$, Figure $4 \mathrm{C}-$ right), but an estimation of the average dendritic length and the average number of branching points can be calculated as follows:

Dendritic length $=(c \times$ distance between line 1 and 2$)$

$+(d \times$ distance between line 2 and 3$)$

$=(9 \times 94 \mu \mathrm{m})+(11 \times 98 \mu \mathrm{m})$

$=1924 \mu \mathrm{m} / 6$ neurons $=321 \mu \mathrm{m} /$ neuron

Branching points $=(c+d) / a=(9+11) / 6=31 / 3$

A

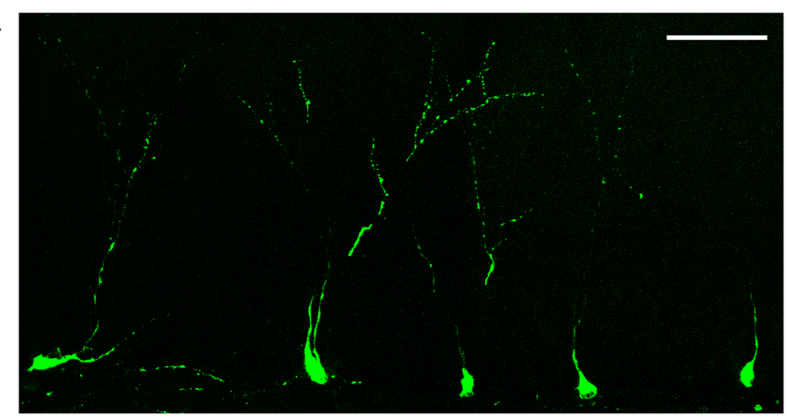

B

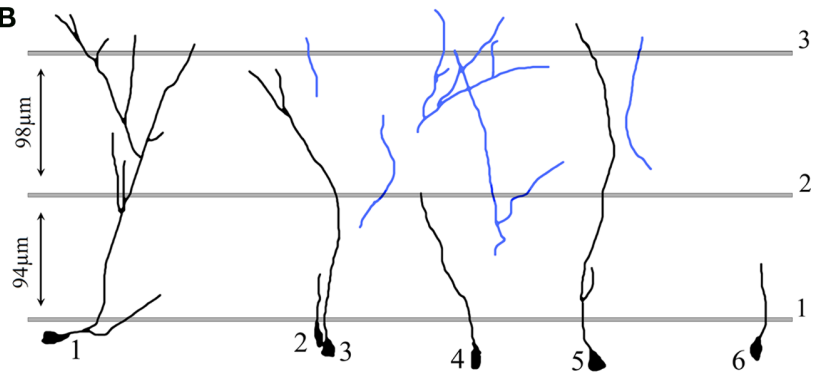

C

\begin{tabular}{|c|c|c|}
\hline \multicolumn{3}{|c|}{ Tracing } \\
\hline Neuron \# & $\begin{array}{c}\text { Dendritic } \\
\text { length }(\mu \mathrm{m})\end{array}$ & $\begin{array}{c}\text { Branching } \\
\text { points }\end{array}$ \\
\hline 1 & 594 & 8 \\
\hline 2 & 38 & 0 \\
\hline 3 & 233 & 1 \\
\hline 4 & 113 & 0 \\
\hline 5 & 251 & 1 \\
\hline 6 & 53 & 0 \\
\hline Avg & 214 & 1.67 \\
\hline
\end{tabular}

Laminar quantification

\begin{tabular}{|cc|}
\hline $\boldsymbol{a}$ & 6 \\
$\boldsymbol{b}$ (line 1) & 7 \\
$\boldsymbol{c}$ (line 2) & 9 \\
$\boldsymbol{d}$ (line 3) & 11 \\
\hline
\end{tabular}

FIGURE 4 | Comparison between dendritic tracing and laminar quantification methods. (A) High-resolution confocal (collapsed stack) image of a section through the DG, showing neurons expressing GFP 14 days post-injection. Scale bar $=60 \mu \mathrm{m}$. (B) Tracings of six GFP-positive dendrites and measurement lines for the quantification of primary (1), secondary (2), and tertiary (3) dendrites. The blue dendrites were considered "disjointed" and were only included in the laminar quantification. (C) Values obtained using both methods.

In both cases the calculated values $(321 \mu \mathrm{m}, 31 / 3)$ are higher than the ones obtained using the tracing method $(214 \mu \mathrm{m}, 1.67$, Figure 4C) due to the additional dendrites that were included in the analysis ("extra" three secondary dendrites and six tertiary dendrites). Still, these values are an underestimation since some dendrites extend beyond line 3 or begin and end between the lines. A higher degree of accuracy can be achieved by adding more measurement lines at shorter intervals.

\section{DISCUSSION}

We have demonstrated here how a series of simple measurements can be used to detect subtle changes in dendritic growth in a subpopulation of adult-born neurons. Quantifying dendrites at vary- 
ing distances from the cell bodies in a disjointed manner enabled us to incorporate many cells and dendrites that would otherwise be excluded from the analysis based on their discontinuous visualization. The result was a comprehensive data set offering an informative representation of the DCX-positive neuronal population in the DGs of studied animals.

\section{ANALYSIS OF THE DATA SET}

Analysis of the rat tissue in experiment 1 revealed an increase in the number of DCX-positive neurons in the treated rat group with no accompanying changes in the ratios representing dendritic branching. This suggests that while the size of the DCX-positive neuronal population was increased, its composition (in terms of the level of development of the neurons comprising it) remained unchanged (Figures 5A,B). This result would be expected when the overall rate of neurogenesis (i.e., production and retention of new neurons) is increased. Such increase can be verified directly using standard immunohistochemical markers. Traditionally, measurements of proliferation and survival of adult-born neurons in the DG are performed using the proliferative marker BrdU and the endogenous Ki-67 (Kee et al., 2002; Wojtowicz and Kee, 2006).

In experiment 2 , the decrease in the ratios representing secondary and tertiary branching $\left(N_{s}\right.$ and $\left.N_{t}\right)$ suggests that a different process is taking place in the KO mice. Not only the size of the DCXpositive neuronal population is changed, but also the relative level of development of the neurons comprising it. In this case, the lack of change in primary sprouting $\left(N_{\mathrm{p}}\right)$ may indicate that only the more mature cells are affected. This result might also suggest that the higher number of DCX-positive cells in the $\mathrm{KO}$ mice is not due to an increase in the rate of neurogenesis, but due to a delay in neuronal

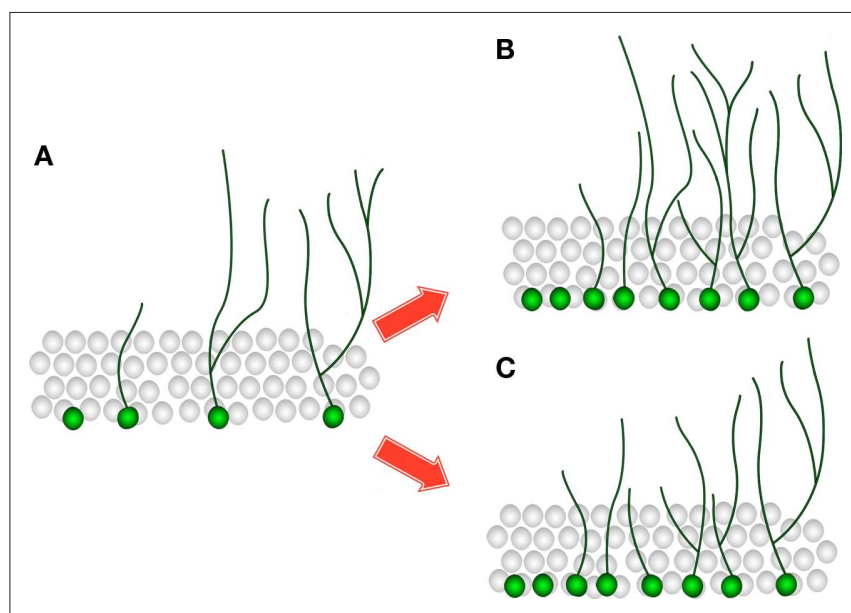

FIGURE 5 | Differential effects on dendritic growth in experiments 1 and 2. In both the treated rat group and KO mice $(\mathbf{B}, \mathbf{C})$ the number of DCX-positive neurons is increased compared to the control rat group and WT mice (A). However, the relative level of development of the neurons comprising the DCX-positive population is unchanged in experiment 1, as evident by a similar portion of cells that have no dendrites, have only primary dendrites or have secondary/tertiary dendrites in the treated group (B) compared to the control group (A). In experiment 2 there is a larger portion of neurons with only primary dendrites and a smaller portion on neurons that have tertiary dendrites in the KO mice (C) compared to the WT mice (A), thus the overall dendritic complexity in the $\mathrm{KO}$ mice is decreased. development which causes neurons to express the marker DCX for a longer period of time (the capacity of the neurons to develop beyond the DCX-expressing stage is diminished). In this case, the "extra" DCX-positive neurons are derived from accumulation of neurons in a relatively early developmental stage - neurons that only have short primary dendrites (Figures 5A,C). This interpretation conforms to the results obtained from the analysis of DCXpositive cells, but other explanations cannot be ruled out without additional complementary experiments. For example, measurements showing that proliferation and/or survival are unchanged (using BrdU and Ki-67) will further support this interpretation. Generally, analysis of DCX-positive cells and dendritic complexity is only one part of the study, and is combined with additional approaches to provide a more comprehensive view (Gao et al., 2007; Wang et al., 2008; Revest et al., 2009).

Thus, laminar quantification of disjointed dendrites effectively detects developmental changes within the DCX-positive neuronal population, but a range of interpretations must be considered for the results and further analysis using additional tools and markers is required in order to place the changes in the context of a general effect on neurogenesis. It should be noted that different experiments were performed on mice and rats. Hence, the different effects observed here are likely due to the experimental design and not necessarily due to species differences.

\section{COMPARISON WITH OTHER METHODS}

One of the main advantages of the method is the use of endogenous markers such as DCX or PSA-NCAM. Several previous studies have attempted to use endogenous markers for dendritic analysis (Rao and Shetty, 2004; Gao et al., 2007; Wang et al., 2008; Revest et al., 2009); however, some problems become apparent when the techniques used in some of those studies are examined in depth. For instance, Revest et al. (2009) included an analysis of dendritic morphology as part of the characterization of adult neurogenesis in a transgenic mouse model. In that study, specific DCX-positive neurons were selected on the basis of their general morphology (i.e., cells that exhibited vertically orientated dendrites that extended into the ML) and minimal overlap with the dendrites of adjacent cells which allowed the authors to unambiguously trace the entire dendritic tree. While this method provided an accurate estimation of the dendritic structure in the analyzed neurons, it is difficult to determine whether the neurons selected for analysis accurately represented the relevant neuronal population.

Another study by Gao et al. (2007) attempted a different approach. They classified the majority of the DCX-positive cells into two developmental stages: A - cells with bipolar short process, located adjacent to the SGZ with the axis of cell body parallel to SGZ, and B - Cells with long dendrites projecting close to or crossing the ML, with axis of cell body perpendicular to SGZ. In this case the relevant neuronal population was more adequately represented, but the resolution of the analysis was low - only two categories of cells were defined, providing minimal data regarding dendritic arborization.

Our intention was to develop a method that achieves both an adequate representation of the entire DCX-positive population and detailed information regarding the length and complexity of the dendritic tree. A direct comparison between our new method and 
the commonly used dendritic tracing method revealed that when the same segment is analyzed by both methods, the data obtained from laminar quantification is comparable in detail and accuracy to the data obtained by tracing, but is more representative of the examined population due to the inclusion of "disjointed" dendrites. Depending on the resolution required for a specific experiment, the new method can be adjusted and additional measurement lines can be inserted to yield better accuracy.

It is interesting to note that while all six neurons in the imaged sample segment were traced for demonstration purposes, most studies using the tracing technique mention the selection of specific neurons for analysis (Ge et al., 2006; Winner et al., 2011). These neurons, chosen at the discretion of the researcher, are considered representative of the studied neuronal population. An examination of the neurons in the sample segment (Figure 4) reveals why this type of subjective selection may pose a problem. While the average length of traced dendrites per neuron was found to be $214 \mu \mathrm{m}$, the lengths of the six individual dendritic trees ranged between 38 and $594 \mu \mathrm{m}$. This variation extended to branching points as well. Therefore, it is likely that an inference based on analysis of specific neurons over others will vary considerably based on the individual neurons that make up the selected cohort.

\section{CRITIQUE}

When using this method it is important to consider its limitations which arise from the basic assumptions the method relies on. For instance, laminar quantification relies on the relatively uniform arrangement of cell bodies along the SGZ and the vertical extension of dendrites toward the ML. This arrangement is only consistent in neurons within a certain range of developmental stages. Therefore, only endogenous markers which are expressed during those stages (and those stages alone) can be used. More advanced neuronal markers are expressed in neurons which have migrated further into the GCL. Once the neurons are no longer arranged along the SGZ it becomes impossible to quantify primary and secondary dendrites in the GCL. However, the basic concept of the method can still

\section{REFERENCES}

Abrous, D. N., Koehl, M., and Le Moal, M. (2005). Adult neurogenesis: from precursors to network and physiology. Physiol. Rev. 85, 523-569.

Ambrogini, P., Cuppini, R., Lattanzi, D., Ciuffoli, S., Frontini, A., and Fanelli, M. (2010). Synaptogenesis in adultgenerated hippocampal granule cells is affected by behavioral experiences. Hippocampus 20, 799-810.

Carlen, M., Cassidy, R. M., Brismar, H., Smith, G.A., Enquist, L.W., and Frisen, J. (2002). Functional integration of adult-born neurons. Curr. Biol. 12, 606-608.

Christie, B. R., and Cameron, H.A. (2006). Neurogenesis in the adult hippocampus. Hippocampus 16, 199-207.

Eadie, B. D., Redila, V. A., and Christie, B. R. (2005). Voluntary exercise alters the cytoarchitecture of the adult dentate gyrus by increasing cellular

be implemented to quantify proximal and distal dendrites in the $\mathrm{ML}$, and this type of analysis may have significant implications for synaptic transmission and connectivity in the DG.

Another important assumption the method relies on is the polarity of young granule neurons. It is important to remember that if the alignment and directionality of the dendrites in the experimental group is affected, the results might not accurately reflect dendritic complexity (because some dendrites will be detected in the "wrong" location or altogether missed).

The use of endogenous markers presents additional limitations. For instance, the expression of DCX persists for up to 3 weeks, thus the exact age of the analyzed cells is unknown. This may become significant in experiments testing the acute effects of certain treatments. Furthermore, an effect on the expression of the endogenous marker itself (e.g., a treatment which reduces the amount of DCX expression in dendrites) might also affect the accuracy of the results.

\section{CONCLUSION}

We have developed an alternative method for measurement and analysis of dendritic growth in a sub-population of DG granule neurons. The main advantage over the pre-existing methods is the improved, unbiased sampling. The method may yield new, alternate data which would be missed with the traditional techniques. Although we focused on the young, adult-generated subpopulation, the technique is also suitable for analysis of other, more mature neuronal populations within the DG. The laminar arrangement of the afferent synaptic inputs suggests specialized functions for each layer of the dendritic tree. Given the dynamic changes within the DG in response to various physiological and pathological manipulations (Jessberger and Gage, 2008; van Praag, 2008; Schaeffer et al., 2009), these functions can now be further examined using dendritic analysis.

\section{ACKNOWLEDGMENTS}

We thank Michael Vu for his help with confocal imaging and analysis. This work was supported by operating grants from CIHR and NSERC.

integration of young newborn dentate gyrus granule cells in the adult hippocampal circuitry. Eur. J. Neurosci. 28, 2381-2392.

Jessberger, S., and Gage, F. H. (2008). Stem-cell-associated structural and functional plasticity in the aging hippocampus. Psychol. Aging 23, 684-691.

Kee, N., Sivalingam, S., Boonstra, R., and Wojtowicz, J. M. (2002). The utility of Ki-67 and BrdU as proliferative markers of adult neurogenesis. J. Neurosci. Methods 115, 97-105.

Meijering, E., Jacob, M., Sarria, J. C., Steiner, P., Hirling, H., and Unser, M. (2004). Design and validation of a tool for neurite tracing and analysis in fluorescence microscopy images. Cytometry A 58, 167-176.

Nguyen, L., Rigo, J. M., Malgrange, B., Moonen, G., and Belachew, S. (2003). Untangling the functional potential of PSA-NCAM-expressing cells in CNS development and brain repair strategies. Curr. Med. Chem. 10, 2185-2196. Nishi, M., Usuku, T., Itose, M., Fujikawa, K., Hosokawa, K., Matsuda, K. I., and Kawata, M. (2007). Direct visualization of glucocorticoid receptor positive cells in the hippocampal regions using green fluorescent protein transgenic mice. Neuroscience 146, 1555-1560.

Overstreet-Wadiche, L. S., Bromberg, D. A., Bensen, A. L., and Westbrook, G. L. (2006). Seizures accelerate functional integration of adult-generated granule cells. J. Neurosci. 26, 4095-4103.

Ramirez-Rodriguez, G., Ortiz-Lopez, L., Dominguez-Alonso, A., Benitez-King, G. A., and Kempermann, G. (2011). Chronic treatment with melatonin stimulates dendrite maturation and complexity in adult hippocampal neurogenesis of mice. J. Pineal Res. 50, 29-37. 
Rao, M. S., Hattiangady, B., AbdelRahman, A., Stanley, D. P., and Shetty, A. K. (2005). Newly born cells in the ageing dentate gyrus display normal migration, survival and neuronal fate choice but endure retarded early maturation. Eur. J. Neurosci. 21, 464-476.

Rao, M. S., and Shetty, A. K. (2004). Efficacy of doublecortin as a marker to analyse the absolute number and dendritic growth of newly generated neurons in the adult dentate gyrus. Eur. J. Neurosci. 19, 234-246.

Redila, V. A., and Christie, B. R. (2006). Exercise-induced changes in dendritic structure and complexity in the adult hippocampal dentate gyrus. Neuroscience 137, 1299-1307.

Revest, J.M., Dupret, D., Koehl, M., FunkReiter, C., Grosjean, N., Piazza, P. V., and Abrous, D. N. (2009). Adult hippocampal neurogenesis is involved in anxiety-related behaviors. Mol. Psychiatry 14, 959-967.

Ruth, R.E., Collier, T. J., and Routtenberg, A. (1982). Topography between the entorhinal cortex and the dentate septotemporal axis in rats: I. Medial and intermediate entorhinal projecting cells. J. Comp. Neurol. 209, 69-78.
Schaeffer, E. L., Novaes, B. A., da Silva, E. R., Skaf, H. D., and MendesNeto, A. G. (2009). Strategies to promote differentiation of newborn neurons into mature functional cells in Alzheimer brain. Prog. Neuropsychopharmacol. Biol. Psychiatry 33, 1087-1102.

Seki, T., and Arai, Y. (1999). Temporal and spacial relationships between PSANCAM-expressing, newly generated granule cells, and radial glia-like cells in the adult dentate gyrus. J. Comp. Neurol. 410, 503-513.

Snyder, J. S., Choe, J. S., Clifford, M. A., Jeurling, S. I., Hurley, P., Brown, A., Kamhi, J. F., and Cameron, $\mathrm{H}$. A. (2009). Adult-born hippocampal neurons are more numerous, faster maturing, and more involved in behavior in rats than in mice. $J$. Neurosci. 29, 14484-14495.

Tronel, S., Fabre, A., Charrier, V., Oliet, S. H., Gage, F. H., and Abrous, D. N. (2010). Spatial learning sculpts the dendritic arbor of adult-born hippocampal neurons. Proc. Natl. Acad. Sci. U.S.A. 107, 7963-7968.

van Praag, H. (2008). Neurogenesis and exercise: past and future directions. Neuromolecular Med. 10, 128-140. van Praag, H., Schinder, A. F., Christie, B. R., Toni, N., Palmer, T. D., and Gage, F. H. (2002). Functional neurogenesis in the adult hippocampus. Nature 415 , 1030-1034.

Wang, J. W., David, D. J., Monckton, J. E., Battaglia, F., and Hen, R. (2008). Chronic fluoxetine stimulates maturation and synaptic plasticity of adultborn hippocampal granule cells. $J$. Neurosci. 28, 1374-1384.

Wang, S., Scott, B. W., and Wojtowicz, J. M. (2000). Heterogeneous properties of dentate granule neurons in the adult rat. J. Neurobiol. 42, 248-257.

Winner, B., Melrose, H.L., Zhao, C., Hinkle, K. M., Yue, M., Kent, C., Braithwaite, A. T., Ogholikhan, S., Aigner, R., Winkler, J., Farrer, M. J., and Gage, F. H. (2011). Adult neurogenesis and neurite outgrowth are impaired in LRRK2 G2019S mice. Neurobiol. Dis. 41, 706-716.

Witter, M. P. (2007). The perforant path: projections from the entorhinal cortex to the dentate gyrus. Prog. Brain Res. $163,43-61$.

Wojtowicz,J.M., and Kee, N. (2006). BrdU assay for neurogenesis in rodents. Nat. Protoc. 1, 1399-1405.

Zhao, C., Teng, E. M., Summers, R. G. Jr., Ming, G. L., and Gage, F. H. (2006).
Distinct morphological stages of dentate granule neuron maturation in the adult mouse hippocampus. J. Neurosci. 26, 3-11.

Conflict of Interest Statement: The authors declare that the research was conducted in the absence of any commercial or financial relationships that could be construed as a potential conflict of interest.

Received: 15 December 2010; accepted: 02 March 2011; published online: 21 March 2011.

Citation: Rosenzweig S and Wojtowicz JM (2011) Analyzing dendritic growth in a population of immature neurons in the adult dentate gyrus using laminar quantification of disjointed dendrites. Front. Neurosci. 5:34. doi: 10.3389/fnins.2011.00034

This article was submitted to Frontiers in Neurogenesis, a specialty of Frontiers in Neuroscience.

Copyright () 2011 Rosenzweig and Wojtowicz. This is an open-access article subject to an exclusive license agreement between the authors and Frontiers Media $S A$, which permits unrestricted use, distribution, and reproduction in any medium, provided the original authors and source are credited. 